\title{
Osteonecrosis as a complication in pediatric patients with acute lymphoblastic leukemia
}

\author{
Ilaria Riccio,1 Elvira Pota,2 Marco Marcarelli,1 Maria Carmen Affinita,2 Daniela Di Pinto,2 \\ Cristiana Indolfi,2 Nicola Del Regno,1 Marco Esposito1 \\ 1Department of Medical and Surgical Specialties and Dentistry, Second University of Naples, Naples; \\ 2Pediatric Oncology Service, Pediatric Department, Second University of Naples, Naples, Italy
}

\begin{abstract}
Osteonecrosis is a significant adverse effect of treatment administered to children suffering from acute lymphoblastic leukemia (ALL) that may have a negative effect on the quality of life. The purpose of this study is to evaluate the rate of secondary vascular osteonecrosis (ON) in a population of pediatric patients with ALL treated with corticosteroids and cytostatic agents. A retrospective analysis of prospectively collected data of the medical records of 328 patients with ALL identified 4 cases with $0 \mathrm{~N}$, corresponding to $1.2 \%$ of all cases observed. Of the 4 patients identified in our study 3 were girls and 1 was a boy, aged from 10 to 16 years old (average age at diagnosis, 12 years). Median time between the diagnosis of ALL and ON was 12.5 months (range, 12 to 36 months). Regarding the lesion size of $\mathrm{ON}$, in all cases the femoral head (monolateral in 1 case and bilateral in 3 cases) was involved and was associated with the scapula-humeral joint in one case. $\mathrm{ON}$ of the weight-bearing joints has been identified as a severe complication in chil-
\end{abstract}

\footnotetext{
Correspondence: Ilaria Riccio, Department of Medical and Surgical Specialties and Dentistry, Second University of Naples, via L. De Crecchio 4, 80138 Naples, Italy.

Tel: +39.081 .5665548 - Fax: +39.081 .5665531 .

E-mail: i.riccio@inwind.it

Key words: Acute lymphoblastic leukemia; Osteonecrosis; Children; Adolescents.

Contributions: all authors equally contributed to the study.

Conflict of interest: the authors declare no potential conflict of interest.

Received for publication: 17 July 2015.

Revision received: 23 November 2015.

Accepted for publication: 19 July 2016.

This work is licensed under a Creative Commons Attribution NonCommercial 4.0 License (CC BY-NC 4.0).

(C) Copyright I. Riccio et al., 2016

Licensee PAGEPress, Italy

La Pediatria Medica e Chirurgica 2016; 38:118

doi:10.4081/pmc.2016.118
}

dren with leukemia that may be associated with the development of articular surface collapse, subsequent debilitating arthritis, sometimes needing arthroplasty. For this reason it is very important to implement prevention strategies, especially in adolescent girls treated with steroids and chemotherapy. An early diagnosis of $\mathrm{ON}$ and careful orthopedic follow-up are necessary in order to avoid bone deformations related to the late start or the wrong therapy.

\section{Introduction}

The latest advances in chemotherapy, along with adequate supportive care, have led to substantial improvements in terms of long-term survival of children with acute lymphoblastic leukemia (ALL), such that the current survival rate is over $80 \% .{ }^{1}$ In contrast, the increased aggressiveness of treatment, which includes the addition of dexamethasone to the standard therapy with prednisone, has caused an increase in complications, such as osteonecrosis (ON) and osteoporosis, both in the medium and long term. ${ }^{2}$ In particular, the increased incidence of osteonecrosis seems to be directly correlated to the use of dexamethasone.3,4 Despite the significant number of scientific papers in the literature, the etiology is still not clear. Probably the pathogenesis of $\mathrm{ON}$ is multifactorial and complex. It includes the suppression of osteoblasts, osteocyte apoptosis, the proliferation and the hypertrophy of intramedullary lipocytes that have negative effects on the nutrient arteries, and may lead to thrombosis and gas emboli. Kenzora et al. have described the theory of cellular stress, in which glucocorticoids were playing a necessary but not sufficient role in the transformation of bone marrow in fat, with the subsequent risk of microembolic phenomena and negative effects on the microcirculation. ${ }^{5}$ Recent studies in vitro have evaluated the impact of glucocorticoids in the pathogenesis of $\mathrm{ON}$ after chemotherapy including the increased glucocorticoid-induced differentiation of mesenchymal stem cells in lipocytes at the expense of osteogenesis. 6,7 Other associated cytotoxic therapies such as methotrexate, asparaginase, and radiotherapy have also been reported as potential risk factors for $\mathrm{ON}$ in patients with cancer not receiving glucorticoids. ${ }^{8}$ Hence, there are actually some controversies about the impact of glucocorticoids in the genesis 
of ON compared to other non-modifiable risk factors such as age $>10$ years, female gender, high body mass index, genetic factors and caucasian race. In regards to the chemotherapy used in ALL patients, the incidence of symptomatic $\mathrm{ON}$ is reported in a variable percentage from 4 to $38 \%$ in retrospective and prospective studies. ${ }^{4,9}$ The symptomatology of $\mathrm{ON}$ after chemotherapy can be extremely variable and switch between a virtual absence of symptoms to a very disabling condition combining pain, loss of musculoskeletal function and variable joint damage. 10 Symptomatic ON after chemotherapy is commonly multifocal and bilateral and involving joints in constant load, such as hip and knee, are generally the most affected and almost never asymptomatic.11 The therapeutic approach to patients with $\mathrm{ON}$ is particularly difficult for both pediatric oncologists and orthopedic surgeons. To date, treatment of ON in ALL children has included supportive care, bed rest, or non-weight bearing exercise, discontinuation of corticoid therapy, and in some cases, surgical intervention. The aim of the present study was to assess the incidence and risk factors relating to $\mathrm{ON}$ in a retrospective series of pediatric patients with ALL diagnosed and treated at the Pediatric Oncology Service at the Second University of Naples.

\section{Materials and Methods}

From January 1982 to December 2003, 328 patients (204 males) aged $<18$ years with newly diagnosed ALL were treated at the Pediatric Oncology Service at the Second University of Naples and enrolled in this study. Patient age at diagnosis ranged from 1 month to 14 years and three months (average age 7 years and two months). According to the prognostic factors the 328 patients were subdivided into three risk categories: i) standard risk (SR): 180 patients (55\%), with absolute leukocyte count $<20.000 / \mu \mathrm{L}$, DNA index between 1.16 and 1.60 , good response to a week of prednisone (peripheral blasts count $<1.000 / \mu \mathrm{L})$, no translocation t $(9 ; 22)$ or t $(4 ; 11)$, blasts count $<5 \%$ in the bone marrow aspirate after the induction therapy; ii) high risk (HR): 99 patients (30\%), with no response to a week of prednisone (peripheral blasts count $>1.000 / \mu \mathrm{L}$ ) or evidence of a translocation $t(9 ; 22)$ or $t(4 ; 11)$ or blasts count $>5 \%$ in the bone marrow aspirate after the induction therapy; iii) intermediate risk (IR): 49 patients (15\%) with characteristics that do not fit into either of the two groups listed above.

According to the consideration if the musculoskeletal manifestations at diagnosis were absent or present, the cohort of patients was further subdivided into 2 groups, as follows.

Group 1: 255 patients (78\%), 163 males and 92 females, with the classic symptoms of the leukemia such as fatigue, pallor, petechiae, fever, purpura, bleeding, and bone pain, but without the musculoskeletal manifestations. Group 2: 73 patients (22\%), 41 males and 32 females, who showed a prevalence of musculoskeletal symptoms or signs, confirmed both upon clinical and radiographic examination.

Among the 328 patients considered, 4 ( 3 females and 1 male) developed $\mathrm{ON}$ corresponding to $1.2 \%$ of all cases.

\section{Results}

\section{Case \#1}

An 11-year-old girl, HR-ALL with poor response to prednisone, was enrolled in the AIEOP-ALL 9502 protocol (overall dosage of drugs: prednisone $2180 \mathrm{mg}$, dexamethasone $123 \mathrm{mg}$, and methotrexate $16022 \mathrm{mg}$ ). At 1 year from the beginning of therapy, the patient began to complain of pain and functional impairment of the left hip with associated limping. At the first physical examination, the patient showed a clear limitation of range of motion (ROM) in all planes. Radiographic examination revealed a standard picture of avascular necrosis of the left femoral head (Figure 1A). Weight-bearing for 30 days was forbidden with the subsequent application of a brace for the weight-bearing limb was prescribed. A rehabilitation program with joint mobilization, muscle strengthening and exercises in open kinetic chain in water was also implemented. A follow-up every 3 months was programmed (Figure 1B). Presently, 9 years from ON diagnosis, clinical examination shows only a light limitation to hip intrarotation, with shortening of the left limb and a satisfactory gait well compensated without the use of any devices. The radiographic examination of the left hip shows a good readjustment reconstruction of the femoral head that appears spherical although it does have a wide and squat neck (Figure 1C).
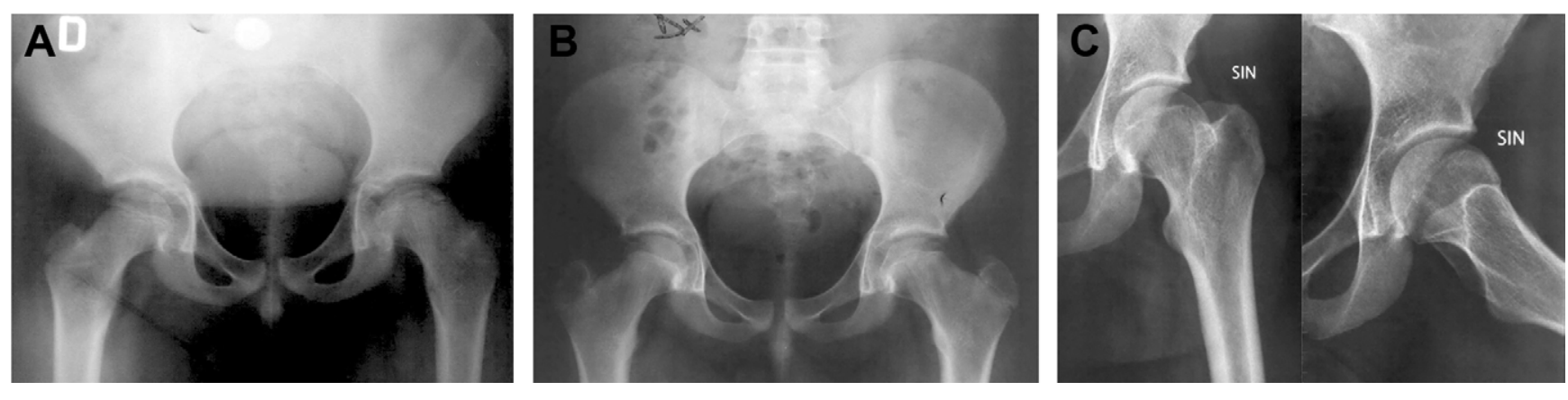

Figure 1. Radiographic examination at eleven years old (A), twelve years old (B), and twenty years old (C) (patient \#1). 


\section{Case \#2}

A 13-year-old boy, IR-ALL, was treated according to the AIEOPALL 2000 protocol (overall dose of drugs: $317.25 \mathrm{mg}$ of dexamethasone, $10934 \mathrm{mg}$ of methotrexate, and $2080 \mathrm{mg}$ of prednisone). After 1 year from beginning therapy the patient presented pain and functional limitation of both hips. Radiographic examination showed the presence of necrosis of both femoral heads. Avoiding load bearing and using the wheel chair together with the previous rehabilitation program were suggested but the patient did not comply despite the clinical and radiographic progression of the disease. For this reason the patient's lower limbs were put in traction for 30 days but the result was not satisfactory due to the patient's lack of compliance to follow the therapy suggested. Currently, about 20 years after the diagnosis of $\mathrm{ON}$, the patient can only stroll around but he is independent. A radiographic examination shows a good osseous remodeling of the femoral heads and the clinical examination shows bilaterally a physiological range of motion.

\section{Case \#3}

The third case concerns a 12-year-old girl, HR-ALL, enrolled into the AIEOP-ALL 9503 protocol (global dosage: $590 \mathrm{mg}$ of dexamethasone, $3802.1 \mathrm{mg}$ of methotrexate, and $5400 \mathrm{mg}$ of prednisone). About three years from diagnosis of ALL, the patient complained of transient pain and functional limitation in her right knee. The clinical examination was negative. The pelvic radiographic examination showed a necrosis of both femoral heads. After about three months, the patient reported the reappearance of pain and functional limitation of both hips. The clinical examination supported by diagnostic imaging led to the diagnosis of bilateral avascular necrosis of the hip. We prescribed absolutely no weight bearing for 60 days and subsequent progressive loading according the tolerance level, and open kinetic chain physical activity with the periodic checks every 3 months. At the last follow-up, 4 years from the $0 \mathrm{~N}$ diagnosis, the clinical examination showed a physiological range of motion of the left hip, while on the right side, there was a light functional limitation in abduction and extrarotation at extreme degrees of movement. The radiographic examination showed a spherical head at the left femur, and a lightly flattened right head with the presence of marginal geodes and osteophytes.

\section{Case \#4}

A 13-year-old girl, HR-ALL, was treated according to the AIEOP- ALL 9503 protocol (4.980 mg of prednisone, $560 \mathrm{mg}$ of dexamethasone and $15,295 \mathrm{mg}$ of methotrexate). After 13 months from diagnosis the patient presented shoulder pain and bilateral coxalgia. The clinical and radiographic examinations were positive for bilateral $\mathrm{ON}$ of the femoral and humeral heads (Figure 2). Absolute rest and the continuative use of a wheel chair associated with physiokinesitherapy (FKT) was planned. After 3 months the partial prohibition of weight bearing and continuative FKT for about 3 years was prescribed. In fact, twelve years from ON diagnosis, a clinical examination showed a slight functional limitation at abduction and extra-rotation of hips at the extreme degree with normal ambulation. The scapula-humeral articulation showed a good range of motion bilater- ally (Figures 3 and 4). A radiographic examination showed a flattened and deformed humeral head at the left shoulder with metaphyseal-epiphyseal areas, geodes and a flattened humeral head with sclerotic areas of the right shoulder. A spherical femoral head, lightly flattened and deformed in some points accompanied by numerous marginal geodes and osteophytes were present in the both hips (Figure 5).

The rehabilitation project initially provided for a period of 1-3 months of discharge with any traction in bed if there was muscle contracture with functional limitation. During this period were conducted massage sessions and active and passive mobilization without causing pain. The rehabilitation continued after this period with recovery exercises of ROM, always maintaining the discharge of the limb with the use of a guardian or sticks Canadians. The mobilization exercises were also prescribed in the pool for patients who could practice them in water without risk for the disease. Patients continued to practice swimming in the maintenance therapy and the stop-therapy.

\section{Discussion}

ON is a potentially disabling complication in both the medium and long term for pediatric patients with ALL. ${ }^{12}$ Recent studies

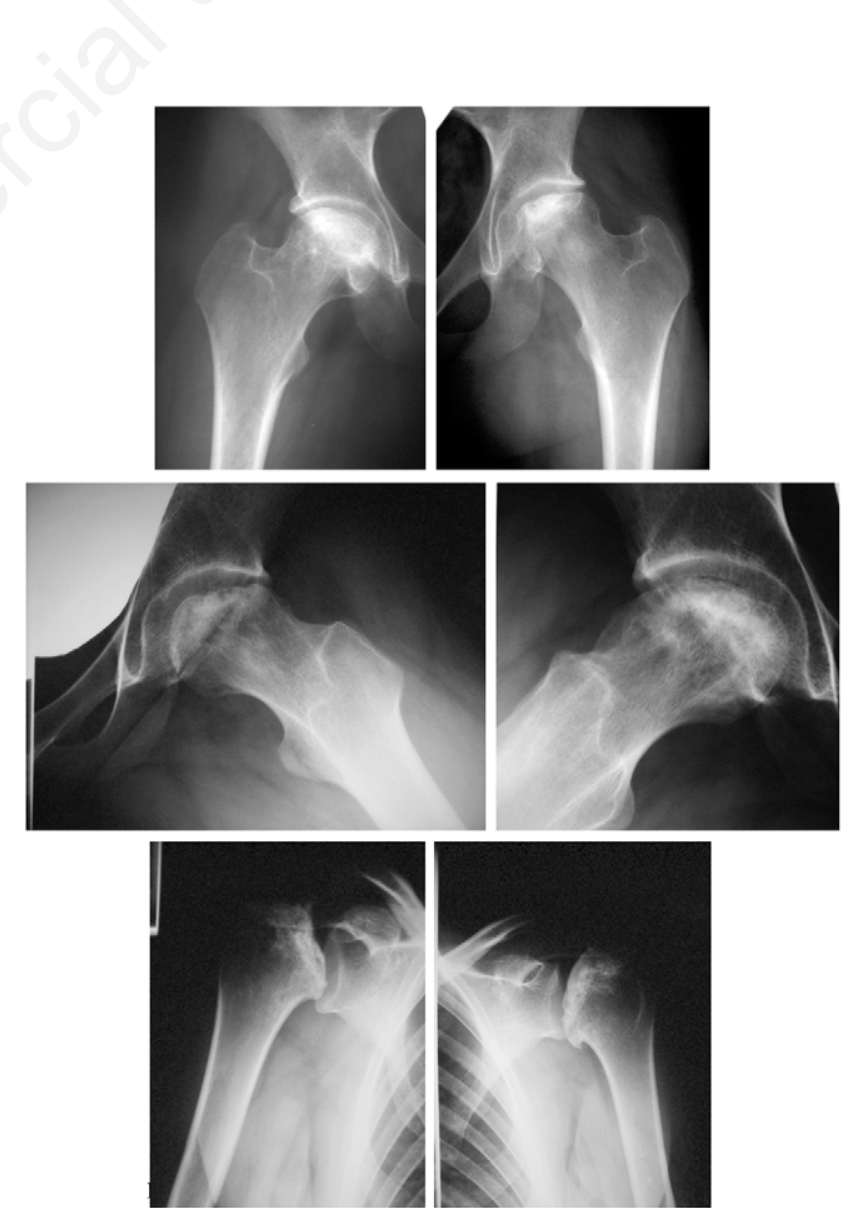

Figure 2. Radiographic examinations at thirteen years old (patient \#4). 
demonstrate a constant and growing attention to these issues and the need to better define the causes and possible correlations with treatment regimens. ${ }^{13}$ It is still unclear the progression of $\mathrm{ON}$, the risk factors and the incidence rate. ${ }^{14} \mathrm{As}$ reported in literature, the overall incidence of symptomatic ON appears to be very variable with percentages ranging from $1 \%$ to $38 \%$; this last value was reported in a study that used magnetic resonance imaging for a systematic screening. 15 The controversy concerning incidence rate appears to be the consequence of retrospective studies.,12 In our study, the overall incidence of ON was $1.2 \%$, although this percentage has to be reinterpreted in relation to the three levels of risk and gender. In fact, the incidence was 0 in the standard-risk patients, $2 \%$ for intermediaterisk patients, and $3 \%$ for patients classified as high-risk. Our incidence of $\mathrm{ON}$ is similar to that observed by Aricò and coworkers in a multicenter study conducted in pediatric patients with ALL. In this study of 1144 patients there were 15 cases of ON (1.1\%). Of these, 12 were female (80\%) and the age of ON diagnosis was found to be ranging from 10 to 16 years in 12 patients, between 6 and 9 years in 2 patients, and below 5 years in 1 patient. Therefore, the incidence was significantly higher in adolescent female patients with a peak of incidence of 7.4\%.16 This consideration was confirmed in our study also even if our cohort of patients was a smaller sample-size. In fact, of the 4 patients with $0 \mathrm{~N}$, three were females (75\%) and all were aged between 11 to 13 years. Therefore these results seem to confirm that the female gender and being $>10$ years represent two main risk factors for the development of $\mathrm{ON}$ in pediatric ALL patients. ${ }^{17}$ Another interesting aspect is the etiology and the possible correlation with the treatment protocols used in childhood ALL. One of the first reports of the possible association between $\mathrm{ON}$ and corticosteroid drugs dates back to an article published in 1957 by Pietrogrande. ${ }^{18}$ Subsequently Felson and Anderson have postulated at least two mechanisms behind the necrosis: the first is represented by direct vascular damage, characterized by a vasculitic process, while the second is gaseous microembolization following hepato-toxicity from corticosteroids. ${ }^{19}$
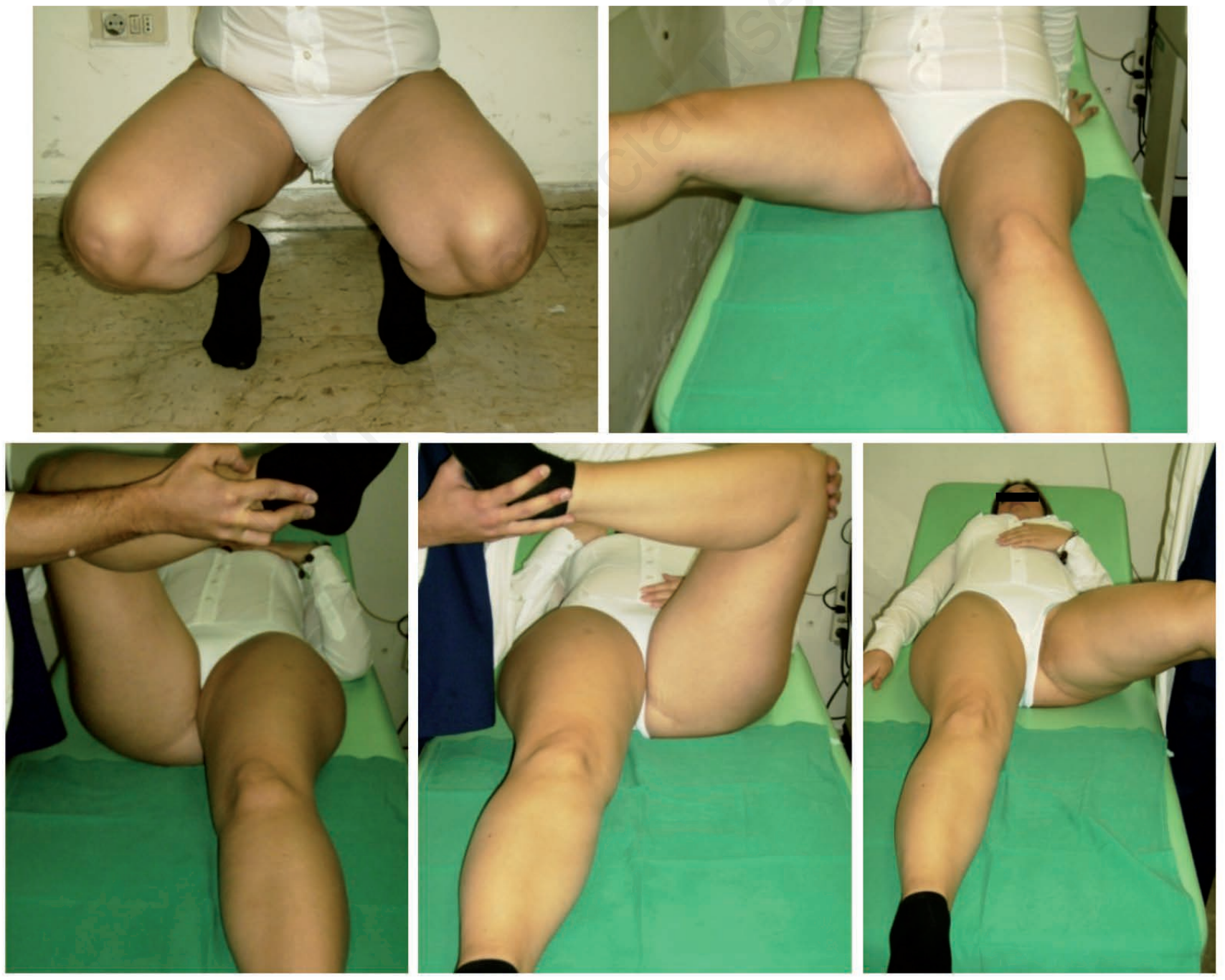

Figure 3. Clinical examination at twenty-three years old at the hips level (patient \#4). 
In particular, the first mechanism of vascular damage could justify a higher incidence of osteonecrosis of the femoral head and load of the humeral head.4,20-22 The high frequency of necrosis at the femur head could be related to the terminal type of vascularization at this level, where any alteration to the blood supply is not compensated by collateral circulation. A similar situation has been reported for the humeral head which presents morphology and vascularization similar to that of the femur, but with a percentage of $\mathrm{ON}$ significantly lower than that of the femur head. This different percentage can be explained by the biomechanics of the hip joint which is subjected to different loads from the shoulder joint. This is confirmed in our study where the femoral head is involved in three cases and the humerus head only in one. Another controversial point of discussion concerns the type of
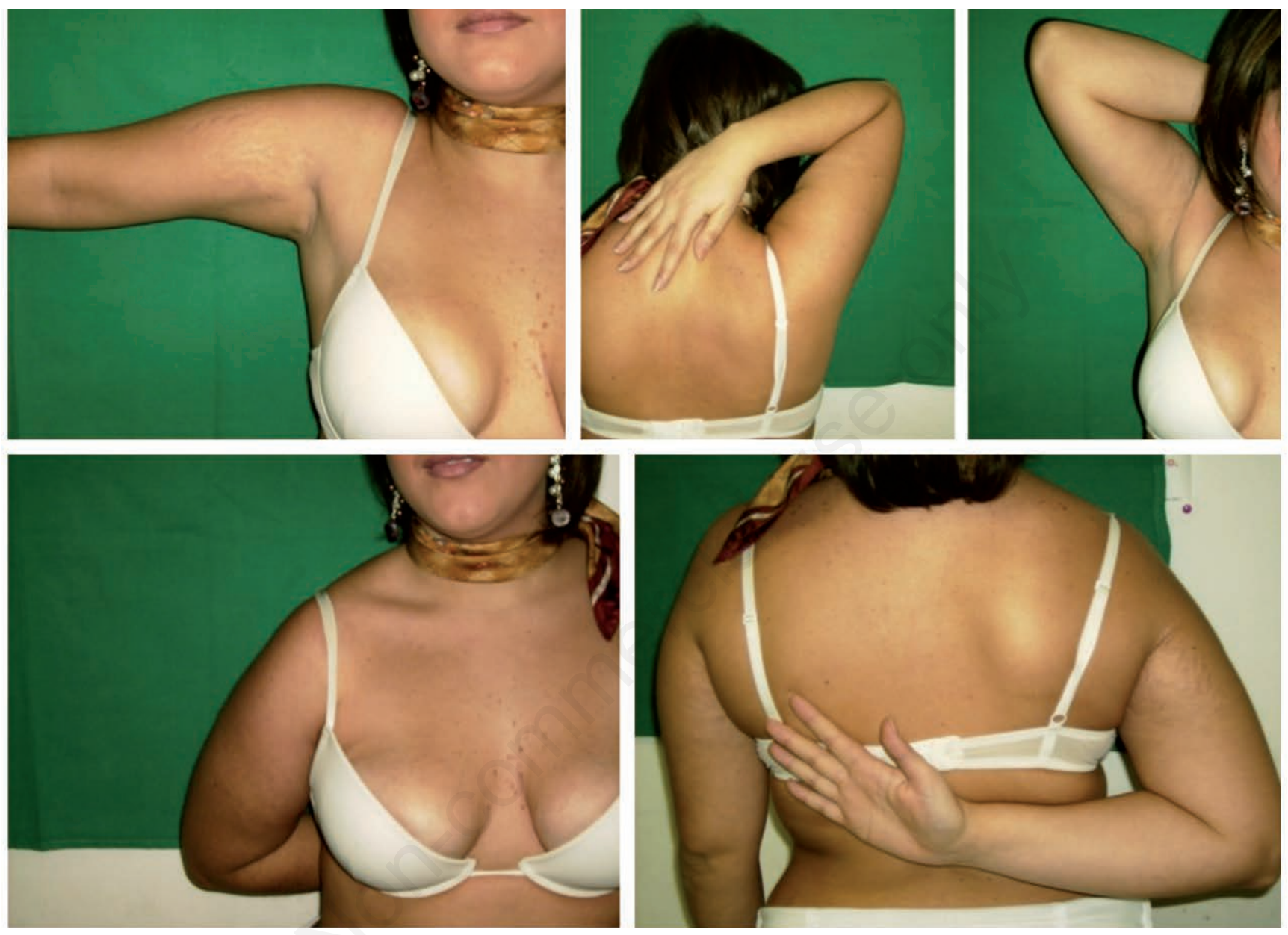

Figure 4. Clinical examination at twenty-three years old at the scapula-humeral articulation level (patient \#4).
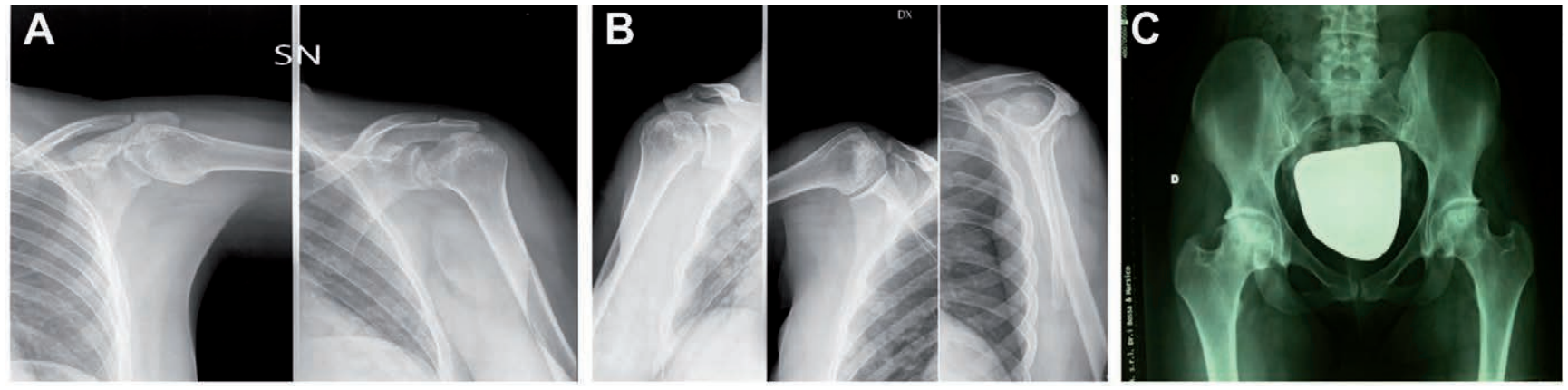

Figure 5. A radiographical examination of the left shoulder (A), the right shoulder (B), and the hips level (C) at twenty-three years old (patient \#4). 
treatment for $\mathrm{ON}$. To date, the variable nature and progression of osteonecrosis secondary to chemotherapy did not lead to the creation of shared orthopedic protocols.

0jala and co-workers have reported a cure rate with restitutio ad integrum in approximately $30 \%$ of patients, ${ }^{9}$ while other multi-center studies have shown that approximately $22-24 \%$ of symptomatic patients have undergone at least one orthopedic surgery. ${ }^{15,23}$ Currently, in severe and rapidly progressive osteonecrosis, a surgical approach is commonly accepted, associated with no weight bearing and physical exercises. In patients with osteonecrosis, smaller lesions and fewer symptoms, the therapeutic approach is generally more conservative, essentially based on the absolute prohibition of loading and active and passive physiotherapy in open kinetic chain. ${ }^{24}$ Conversely, Jager and coworkers support the importance of early surgery in order to delay as long as possible or avoid arthroplasty. ${ }^{25}$ Early surgery can certainly be a valuable aid to rebuild the joint deformed. It uses essentially the femoral or pelvic osteotomies and in selected cases hemi-resurfacing arthroplasty of the femoral head. ${ }^{26}$ Conversely, arthrodesis is absolutely contraindicated in patients who have multiple joints involved.27 Adult and pediatric studies have also suggested the use of bisphosphonates such as alendronate or pamidronate as part of the medical management of $\mathrm{ON}$ to delay bone collapse.28,29 The treatment protocol adopted in our patients was essentially a conservative approach based on the prohibition of load and physiotherapy initially in open kinetic chain. Our results seem to confirm the validity of conservative treatment without surgical intervention in pediatric patients. Detection of lesions at an early asymptomatic stage affords opportunities for prompt enrollment onto clinical trials of interventions to prevent progressive joint damage. Recently, Kaste et al showed the utility of early screening magnetic resonance imaging for extensive hip osteonecrosis in pediatric patients treated with glucocorticoids. Of 498 patients studied, extensive asymptomatic osteonecrosis was identified by early screening in 26 patients (41 hips). ${ }^{30}$ In other study Sansgiri et al. showed that the some MRI signal abnormalities predict the development of osteonecrosis earlier. Of 481 patients, the authors identified 21 cases with subtle poorly defined geographically delineated MRI signal abnormalities in knees or hips, or both, that progressed over a median of 4 months to florid MRI signs of osteonecrosis. ${ }^{31}$

\section{Conclusions}

The therapeutic success in the treatment of pediatric ALL can be partly explained by intense chemotherapy, which, however, increases the risk of side effects. Osteonecrosis is well known as being one of these complications. ON can be a very disabling condition with, sometimes, a complete replacement of the involved joint. For this reason, periodic orthopedic check-ups, especially in female patients $>10$ years of age, are essential in the surveillance protocol in order to avoid delays in ON diagnosis and the wrong therapies that may worsen the evolution of the disease over time, thus requiring surgical intervention.

\section{References}

1. Kamps WA, van der Pai-De Bruin KM, Veerman AJ, et al. Long-term results of Dutch Childhood Oncology Group studies for children with acute lymphoblastic leukemia from 1984 to 2004. Leukemia 2010;24:309-13.

2. Barr RD, Sala A. Osteonecrosis in children and adolescents with cancer. Pediatr Blood Cancer 2008;50:483-5.

3. Mattano L, Sather H, La M, et al. Modified dexamethasone reduces the incidence of treatment related osteonecrosis in children and adolescents with high risk acute lymphoblastic leukemia: a report of COG-1961. Blood 2003;102:221a (Abstract).

4. Kawedia JD, Kaste SC, Pei D, et al. Pharmacokinetic, pharmacodynamic, and pharmacogenetic determinants of osteonecrosis in children with acute lymphoblastic leukemia. Blood 2011;117:2340-7.

5. Kenzora JE. Treatment of idiopathic osteonecrosis: the currentphilosophy and rationale. Orthop Clin N Am 1985;16:717-25.

6. Motomura G, Yamamoto T, Miyanishi K, et al. Bone marrow fat-cell enlargement in early steroid-induced osteonecrosis histomorphometric study ofautopsy cases. Pathol Res Pract 2005;200:807-11.

7. Lee JS, Roh HL, Kim CH, et al. Alterations in the differentiation ability of mesenchymal stem cells in patients with nontraumatic osteonecrosis of the femoral head: comparative analysis according to the risk factor. J Orthop Res 2006;24:604-9.

8. Murphy RG, Greenberg ML. Osteonecrosis in pediatric patients with acute lymphoblastic leukemia. Cancer 1990;65:1717-21.

9. Ojala AE, Lanning FP, Paakko E, Lanning BM. Osteonecrosis in children treated for acute lymphoblastic leukemia: a magnetic resonance imaging study after treatment. Med Pediatr Oncol 2001;29:260-65.

10. Strauss AJ, Su JT, Dalton VM, et al. Bony morbidity in children treated for acute lymphoblastic leukemia. J Clin Oncol 2001;19:3066-72.

11. Group CO. Symptomatic multifocal osteonecrosis. A multi center study. Clin Orthop Relat Res 1999;369:312-26.

12. Winkel ML, Pieters R, Hop WC, et al. Prospective study on incidence, risk factors, and long-term outcome of osteonecrosis in pediatric acute lymphoblastic leukemia. J Clin Oncol 2011;29:4143-50.

13. Kerachian MA, Séguin C, Harvey EJJ. Glucocorticoids in osteonecrosis of the femoral head: a new understanding of the mechanisms of action. Steroid Biochem. Mol Biol 2009;114:121-8.

14. Karimova EJ, Wozniak A, Wu J, et al. How does osteonecrosis about the knee progress in young patients with leukemia? A 2- to 7-year study. Clin Orthop Relat Res 2010;468:2454-9.

15. Ojala AE, Paakko E, Lanning FP, et al. Bone marrow changes on MRI in children with acute lymphoblastic leukaemia 5 years after treatment. Clin Radiol 1998;53:131-6.

16. Aricò M, Boccalatte MFP, Silvestri D, et al. Osteonecrosis: an emerging complication of intensive chemotherapy for child- 
hood acute lymphoblastic leukaemia. Haematologica 2003; 88:747-53.

17. Hogler W, Wehl G, van Staa T, et al. Incidence of skeletal complications during treatment of childhood acute lymphoblastic leukemia: comparison of fracture risk with the General Practice Research Database. Pediatr Blood Cancer 2007;48:21-7.

18. Pietrogrande V, Monteleone M, Mollica Q. Natura e aspetti dell'osteopatia leucemica. Ort Traum App Mot 1960;28:383-7.

19. Felson D, Anderson J. A cross-study evaluation of association between steroid dose and bolus steroids and avascular necrosis of bone. Lancet 1987;1:902-6.

20. Janke LJ, Liu C, Vogel P, et al. Primary epiphyseal arteriopathy in a mouse model of steroid-induced osteonecrosis. Am J Pathol 2013;183:19-25.

21. Wei SY, Esmail AN, Bunin N, et al. Avascular necrosis in children with acute lymphoblastic leukemia. J Pedatr Orthop 2000;20:331-5.

22. Sala A, Mattano LA Jr, Barr RD. Osteonecrosis in children and adolescents with cancer: an adverse effect of sistemi therapy. Eur J Cancer 2007;43:683-9.

23. Burger B, Beier R, Zimmermann M, et al. Osteonecrosis: a treatment related toxicity in childhood acute lymphoblastic leukemia (ALL) - experiences from trial ALL-BFM 95. Pediatr Blood Cancer 2005;44:220-5.
24. Hungerford DS, Jones LC. Asymptomatic osteonecrosis: should it be treated? Clin Orthop Relat Res 2004;429:124-30.

25. Jäger M, Begg MJ, Ready J, et al. TS. Primary total hip replacement in childhood, adolescence and young patients: quality and outcome of clinical studies. Technol Health Care 2008;16:195-214.

26. Jäger M, Begg MJ, Krauspe R. Partial hemi-resurfacing of the hip joint - a new approach to treat localosteochondral defects? Biomed Tech 2006;51:371-6.

27. Westhoff B, Jäger M, Krauspe R. Osteonecrosis after chemotherapy in children. Orthopäde 2008;37:56-62.

28. Nishii T, Sugano N, Miki H, et al. Does alendronate prevent collapse in osteonecrosis of the femoral head? Clin Orthop Relat Res 2006;443:273-9.

29. Leblicq C, Laverdière C, Dècarie JC, et al. Effectiveness of pamidronate as treatment of symptomatic osteonecrosis occurring in children treated for acute lymphoblastic leukemia. Pediatr Blood Cancer 2013;60:741-7.

30. Kaste SC, Pei D, Cheng C, et al. Utility of early screening Magnetic Resonance Imaging for extensive hip osteonecrosis in pediatric patients treated with glucocorticoids. J Clin Oncol 2015;33:610-5.

31. Sansgiri RK, Neel MD, Soto-Fourier M, et al. Unique MRI findings as an early predictor of osteonecrosis in pediatric acute lymphoblastic leukemia. AJR 2012;198:432-9. 